\title{
Une charte de collaboration - sans collaboration?
}

Jürg Schlup

Président de la FMH

\section{Collaboration entre professionnels de la santé}

La collaboration interprofessionnelle va sans aucun doute gagner en importance dans le domaine de santé. En effet, une bonne prise en charge médicale demandera à l'avenir davantage de coordination, car les connaissances en médecine ne cessent de croître, impliquant toujours plus de spécialisation. Cet élargissement des compétences ouvrira la porte à de nouvelles professions de la santé tandis que les professions existantes évolueront. L'augmentation des temps partiels ou le travail dans des centres de soins imposera par ailleurs des exigences plus élevées en termes de collaboration.

\section{A aucun moment, les représentants légitimes des groupes professionnels n'ont été impliqués dans la phase d'élaboration de la charte ni dans les possibilités de modification accordées ultérieurement.}

Correspondance:

FMH

Dr Jürg Schlup

Elfenstrasse 18

CH-3000 Berne 15

Tél. 0313591111

Fax 0313591112
La charte «Collaboration entre les professionnels de la santé» de l'ASSM aborde donc un sujet essentiel et d'une importance majeure pour la qualité des soins et la motivation au travail des médecins et professionnels de la santé. La collaboration interprofessionnelle au quotidien a donné lieu à des expériences très positives. La recherche révèle, elle aussi, que la Suisse obtient au plan international de très bons résultats dans la collaboration entre le corps médical et les soignants [1].

\section{Evolution de la collaboration interprofession- nelle: exigences et réalité}

Améliorer et développer ce qui est bien est judicieux.

L'élaboration d'une charte entendant «refléter l'esprit de coopération des professionnels de la santé» aurait reçu le soutien total de la FMH si elle avait été initiée par des représentants légitimes des groupes professionnels auxquels elle s'adresse. Mais le fait que cette charte ait été rédigée par un groupe, dont la plupart des membres ne possède aucune légitimité sensiblement la portée. La représentation très hétérogène des professions de la santé au sein de ce groupe de travail - certaines mêmes y étant complèdémocratique au sein de leur profession, en réduit tement absentes, comme les chiropraticiens, les sages-femmes ou les assistantes médicales - affaiblit fortement le droit à une «collaboration interprofessionnelle» telle que réclamée dans la charte.

Au-delà de l'élaboration de la charte, les possibilités de modification accordées ultérieurement n'ont pas non plus permis une véritable implication de représentants légitimes des groupes professionnels: la première version a été commentée par des «experts choisis». Une large consultation a précédé la deuxième version mais le fait qu' «un certain scepticisme était néanmoins perceptible au sein du corps médical» et que de «nombreuses institutions» souhaitaient «être davantage impliquées dans l'élaboration de cette charte» n'a pas incité l'ASSM à modifier son texte.

\section{Evolution de la collaboration interprofes- sionnelle: légitimité et transparence comme conditions essentielles}

Le processus d'élaboration de la charte montre qu'il aurait été plus judicieux de fixer tout d'abord les règles du jeu pour développer en commun la «collaboration entre les professionnels de la santé». Un tel code devrait par exemple intégrer les groupes d'intérêts, montrer la transparence des processus de sélection et tenir compte de la critique. Il conviendrait également de soulever la question du poids à accorder aux experts par rapport aux représentants légitimes des groupes professionnels.

La FMH approuve que le développement commun de directives interprofessionnelles revienne aux associations et organisations professionnelles. En revanche, elle rejette que la charte édicte à l'avance les contenus souhaités ou souhaitables.

\section{Collaboration entre professionnels de la} santé: qu'en est-il du contact avec le patient? Même les patients, qui pourtant forment le groupe d'intérêts le plus important et de loin le plus nombreux, n'ont pas été impliqués dans l'élaboration de la charte - contrairement aux «éléments clés et obligations» qu'elle décrit. Ni le groupe de travail, ni le conseil d'experts, ni la liste des personnes conviées à la consultation ni celle des participants au Symposium sur la charte ne comptent un représentant des patients.

Bien que la charte doive «optimiser le traitement $d u$ patient», ce dernier n'a été à aucun moment pris en compte dans le processus d'élaboration du docu- 
ment et de ses contenus. Reste à savoir dans quelle mesure le patient aurait à l'avenir un interlocuteur responsable de son traitement si les points centraux de la charte - comme la répartition des tâches et des responsabilités entre les groupes professionnels «selon la situation» - étaient mis en œuvre. On peut également se demander qui serait tenu responsable visà-vis du patient en cas de dommage, au vu de la souplesse des réglementations et du chevauchement des domaines de responsabilité. Le médecin perd son rôle de responsable thérapeutique, chargé de coordonner le traitement pour le bien du patient, sans qu'un nouvel interlocuteur ne soit désigné.

\section{Une nouvelle répartition des responsabilités} au détriment de tous les participants?

Les points évoqués précédemment sur la responsabilité, qui devrait être "assumée selon les compétences professionnelles», constituent le problème central de la charte. Cette notion de "responsabilité», n'étant pas assez différenciée, ni juridiquement explicite, concrètes» compte tenu du nombre infini de situations, les prises de décision ponctuelles avec une responsabilité partielle manquent de clarté et fragmentent le traitement. Par ailleurs, la répartition de la responsabilité face au patient engendre une insécurité juridique pour les médecins et les professionnels de la santé concernés, principalement dans le domaine ambulatoire. La responsabilité ne reviendrait plus systématiquement au médecin, mais à une autre personne selon la situation et l'institution de soins. Les traitements normaux mais aussi les cas de divergences entre les professionnels, les cas de dommages ou de litiges juridiques deviendraient compliqués en renonçant à un seul interlocuteur explicite, chargé de déléguer les tâches.

\section{La charte - un document dynamique?}

Le processus d'élaboration de la charte, décrit précédemment et dont les organisations de médecins ont pratiquement été exclues, enlève sa crédibilité au caractère dynamique annoncé de ce document. Pour

\section{Le médecin perd son rôle de responsable thérapeutique, chargé de coordonner le traitement pour le bien du patient, sans qu'un nouvel interlocuteur ne soit désigné.}

laisse la place à différentes interprétations. L'orientation générale est cependant clairement perceptible, il s'agit d'une "prise de responsabilités supplémentaires» par certaines professions de la santé, concrétisant ainsi un élargissement des «compétences pour le diagnostic, la prescription et la facturation». La volonté de suivre «des critères hiérarchiques et/ou des profils professionnels traditionnels» est rejetée au bénéfice d'une conception de gestion en fonction de la situation. Les directives élaborées pour «les situations concrètes, dans un processus de concertation commun» doivent en partie s'appliquer à l'organisation des soins en général et à chaque institution en particulier.

La charte fait l'impasse sur le fait que les médecins sont aujourd'hui déjà "prêts à déléguer des tâches selon la situation», ce qu'ils font déjà dans le cadre du modèle bien connu de la délégation. Ce modèle, basé sur la compétence médicale, présente l'avantage de pouvoir attribuer, de manière explicite et à tout moment, la responsabilité de tout ce qui doit être fait ou pas. Compte tenu de la spécialisation croissante et de la multiplication des professions de santé, ce modèle présente également l'avantage de centraliser tous les éléments du traitement et de garantir les compétences requises - ce qui accroît la qualité du traitement et évite les doublons.

S'il semble difficile de préciser la «définition des compétences et des responsabilités dans des situations la FMH, la participation active à un «processus d'évolution et de transformation» ne consiste pas à pouvoir prendre de temps en temps position, et ensuite à devoir attendre que certaines critiques donnent éventuellement lieu à de petites modifications.

A l'inverse du caractère évolutif ou dynamique qu'elle annonce, la charte fixe déjà une mise en œuvre très concrète de ses contenus. Plutôt que de préférer un développement de fond issu d'une révision par des représentants légitimes, il a déjà été prévu que la charte soit un «outil d'enseignement» et serve de base «pour le contact institutionnalisé avec des associations»; et qu'un monitoring soit organisé en vue de procéder, «le cas échéant», à des adaptations au bout de deux ans. Enfin, dans sa mise en œuvre, la charte oublie que l'engagement commun des associations pour adapter les lois, les ordonnances et les tarifs implique une recherche préalable de consensus. Au vu du déroulement de la phase d'élaboration et des contenus controversés, la charte ne sera pas à la hauteur du rôle qu'elle s'est donné.

\section{Référence}

1 Heinen, $\mathrm{M} \mathrm{M}$ et al. Nurses' intention to leave their profession: A cross sectional observational study in 10 European countries. International Journal of Nursing Studies. 2013;50:174-84. 